\title{
Combined effect of boundary layer formation and surface smoothing on friction and wear rate of lubricated point contacts during normal running-in processes
}

\author{
Yazhao ZHANG, Alexander KOVALEV, Yonggang MENG* \\ State Key Laboratory of Tribology, Tsinghua University, Beijing 100084, China \\ Received: 05 April 2018 / Revised: 26 June 2018 / Accepted: 29 June 2018 \\ (C) The author(s) 2018. This article is published with open access at Springerlink.com
}

\begin{abstract}
The combined effect of boundary layer formation and surface smoothing on friction and wear rate of metallic surfaces under lubricated point contact condition was investigated. The double trend of friction coefficient variations was revealed during running-in and sub-running-in processes. The evolution of surface topography was measured on-site using white-light interference profilometer and analyzed using bearing area curves. Comprehensive theoretical equations that explicitly express the contributions of boundary friction, adhesive friction and wear have been derived, and results obtained by these equations were compared with experimental observations. It is concluded that the theoretical models are quantitatively adequate to describe the combined effect of surface smoothing due to mechanical wear and formation of boundary films on the changes in friction and wear rate during normal running-in processes.
\end{abstract}

Keywords: running-in; bearing area curves; friction modeling; wear modeling; mixed lubrication

\section{Introduction}

Changes in friction and wear during most machine operations typically occur immediately after the onset of sliding in the interface between contacting fresh dry or lubricated solid surfaces. The change is apparently associated with the transitions of microscopic contact and lubrication states arisen during sliding process. The initial transition is known as "running-in" or "break-in" process that occurs under nonzero normal force and relative sliding [1]. So far, very little is known about the running-in phenomenon. Most of the previous studies conducted on the friction and wear behavior of materials overlooked the initial transitional stage in machine operations while focused on the steady phase that follows the running-in process. However, Blau [2] highlighted that owing to ignoring the running-in aspects of sliding, researchers overlooked important clues associated to the evolution of conjoint process that later affects the long-term machine operation. Thus, better understanding of the running-in process is essential for efficient machine operations.

It is well known that the running-in behavior is induced by wear, and wear prediction is the most significant tribological challenge [3]. The transformation of metallic surface caused by wear is a complex phenomenon that involves the following cyclic changes $[4,5]$ when the tribopair operates under the appropriate conditions: (1) plastic deformation develops a highly deformed and oriented hardened sub-surface layer; (2) micro-cracks form within the hardened layer; (3) owing to the coalescence of micro-cracks, wear particles are generated and the remaining plateaus bear the normal load; (4) plateau fails leaving a smoother surface that restart the wear cycle. This scenario is suitable for both the running-in and steady wear stages, and it also fits the transitional "sub-stage"

* Corresponding author: Yonggang MENG, E-mail: mengyg@tsinghua.edu.cn 


\begin{tabular}{|llll|}
\hline \multicolumn{2}{|l|}{ List of symbols } & & \\
$A_{h}$ & Fluid film area & $\dot{V}_{0}$ & Initial value of $\dot{V}$ \\
$h$ & Oil film thickness & $\dot{V}_{s}$ & Steady value of $\dot{V}$ \\
$p_{h}$ & Hydrodynamic pressure & $W$ & Total load \\
$u$ & Sliding velocity & $W_{b}$ & Load fraction supported by boundary layer \\
$F$ & Total friction force & $W_{h}$ & Load fraction supported by hydrodynamic \\
$F_{b}$ & Solid-boundary layer-solid friction force & & lubricant \\
$F_{h}$ & Viscous shear force & $W_{s}$ & Load fraction supported by real solid-solid \\
$F_{s}$ & Solid-solid contact adhesive friction force & & contact areas \\
$f$ & Total friction coefficient & $\alpha$ & Hydrodynamic force fraction, $W_{h} / W$ \\
$f_{0}$ & Initial value of COF in the continuous test & $\alpha_{0}$ & Initial value of $\alpha$ \\
$f_{0}^{\prime}$ & The value of COF at $\alpha=\alpha_{0}, \beta=\beta_{s}$ & $\alpha_{s}$ & Steady value of $\alpha$ \\
$f_{s}$ & Steady value of COF in the continuous test & $\beta$ & Boundary layer force fraction, $W_{b} /\left(W-W_{h}\right)$ \\
$f_{s}^{\prime}$ & The value of COF at $\alpha=\alpha_{s}, \beta=\beta_{0}$ & $\beta_{0}$ & Initial value of $\beta$ \\
$H$ & Material hardness & $\beta_{s}$ & Steady value of $\beta$ \\
$K$ & Wear coefficient & $\bar{\beta}_{3}$ & Mean value of $\beta$ for each interval \\
$k_{\alpha}$ & Rate constant relating to the surface & $\dot{\gamma}$ & Shear rate \\
& smoothing & $\eta$ & Viscosity of lubricant \\
$k_{\beta}$ & Rate constant relating to the formation of & $\mu_{b}$ & Boundary friction coefficient \\
& boundary layer & $\mu_{h}$ & Limiting shear stress coefficient \\
$s$ & Sliding distance & $\mu_{s}$ & Solid-solid contact friction coefficient \\
$V$ & Wear volume & $\tau_{h}$ & Viscous shear stress \\
$\dot{V}$ & Wear rate & $\tau_{\text {lim }}$ & Limiting shear stress \\
\hline \hline
\end{tabular}

between these phases. The main aim of wear prediction is to estimate the material loss depending on the mechanical properties and surface parameters of rubbing solids as well as the certain schemes of tribotesting devices used. Owing to the multi-scaled physical factors affecting friction and wear, numerous wear models have been proposed [6,7]. Meng and Ludema [8] were the first to summarize and classify the previously proposed wear models in 1995. They distinguished three main types of wear equations: empirical, phenomenological and those based on selected failure mechanisms. Many of the existing wear models employed to calculate the material loss are based on Archard wear law [9] that is widely used in engineering calculations. It is a quantitatively simple equation expressing the wear volume generated in the sliding process as a linear function of sliding distance and normal load. Archard's law is applicable for the steady wear stage; however, it does not apply to the running-in process, during which the wear rate is usually not constant.

The prediction of variation in friction coefficient during the running-in process is also challenging. The friction force in lubricated contacts is composed of boundary friction at asperity contacts and viscous shear resistance of fluid lubricant films. According to Bowden and Tabor's theory [10], the boundary friction is further divided into three components, solid-solid contact adhesive friction, solid-boundary layer-solid contact friction and the plowing force caused by the asperity interlocking between the contacting surfaces and indentation of harder asperities into the softer counterparts. During a normal running-in process, contacting asperities could continuously become more or less smoothened with increasing running distance owing to the wear and plastic deformation. Meanwhile, adsorption film and/or tribochemical surface reaction film could form at the asperities [11], leading to variation in friction coefficient even under constant load and sliding speed condition. The interplay and 
combined effect of surface smoothing and boundary layer formation complicate the running-in process, leading to more complex model.

The aim of this study is to investigate and to distinguish the combined effect of boundary film formation and surface smoothing on the changes in coefficient of friction (COF) and wear rate during the running-in process. A series of start-stop ball-onplate experiments were performed to separate the boundary layer effect from the evolution of surface topography, which was measured on-site repeatedly and interruptedly using an optical interference profilometer. The bearing area curve was used to calculate the material losses at different running distances, and the wear rate vs. sliding distance curve was obtained. Comprehensive theoretical equations have been derived based on Bowden and Tabor's boundary lubrication theory and Archard's wear equation. The equations explicitly express the contributions of boundary film and surface smoothing effects to $\mathrm{COF}$ and wear rate during the running-in process. The obtained results were compared to experimental observations to verify the accuracy of the developed model.

\section{Correlation between wear and friction during running-in processes}

Wear has a number of different definitions that describe the progressive damage of surface caused by relative motion with respect to another substance [12]. Two wear processes accompany the surface damage caused by friction. One process involves the loss of materials, the other is the change in the surface topography as a result of plastic deformation, both of which occur in the sliding lubricated surfaces and affect the friction force acting on the interface [13]. Figure 1 shows the typical pattern of variation in wear volume in relation to the sliding distance in tribology tests and the accompanying friction response of the sliding lubricated surfaces. The pattern represents different wear sequences that occur during friction tests $[12,14]$ and it does not depend on the type of material, test condition and the selected triboscheme.

According to the common theory of wear transition [1], during a normal running-in stage (Region A in
Fig. 1), both wear rate and friction coefficient decrease with the sliding distance. Then the wear process gradually transfers to a steady state (Region B in Fig. 1) as the change in the surface topography is stabilized. When the steady wear characterized by a constant wear rate surpasses a certain period, severe wear occurs at the interface, steadily increasing the wear rate and the friction coefficient during the so-called severe wear stage (Region C in Fig. 1).

In contrast to the uniform wear pattern, the pattern of accompanying friction behavior is multiform during the wear process. Blau has distinguished eight main patterns of friction force that could occur during the running-in process [1]. Several irreversible changes simultaneously take place in the sliding contacting surfaces, sub-surface areas and interfacial regions, resulting in various time-dependent variations in friction coefficient. The pattern of friction coefficient curve shown in Fig. 1 corresponds to the normal situation often taken place under typical lubricated conditions [1,12], which will be discussed in Section 2.1.

\subsection{Estimation of friction force under lubrication condition}

As the plowing force is assumed to be negligible in certain circumstances, the total friction force $F$ under lubricated condition can be simply expressed as the sum of three components, $F_{h}, F_{b}$ and $F_{s}$, as follows,

$$
F=F_{h}+F_{b}+F_{s}
$$

where $F_{h}$ denotes the viscous shear force of fluid lubricant films at interface, $F_{b}$ is the solid-boundary

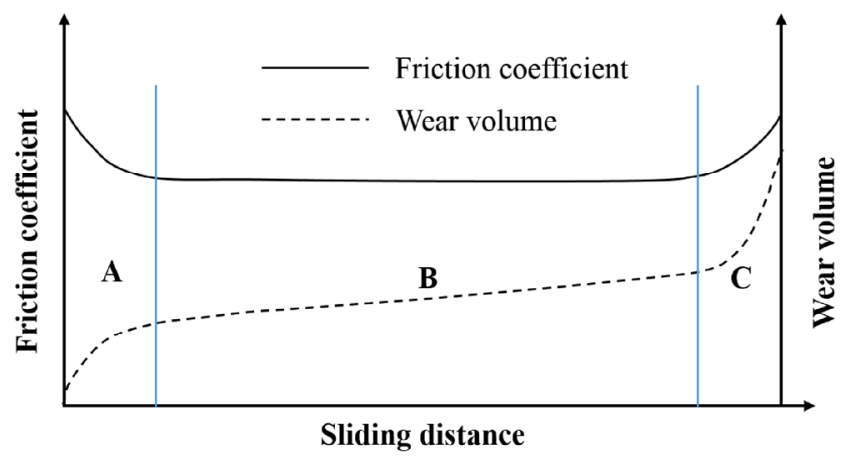

A: Running-in; B: Steady state; C: Severe wear

Fig. 1 Sketch of a typical correlation between wear and friction. 
layer-solid friction force, and $F_{s}$ is the solid-solid contact adhesive friction force (see Fig. 2).

The viscous shear force $F_{h}$ is the integration of the viscous shear stress $\tau_{h}$ over the fluid film area $A_{h}$.

$$
F_{h}=\int_{A_{h}} \tau_{h} \mathrm{~d} A=\int_{A_{h}} \frac{\eta u}{h} \mathrm{~d} A=\int_{A_{h}} \eta \dot{\gamma} \mathrm{d} A
$$

where $\eta$ is the viscosity of lubricant under contacting pressure and temperature, $u$ is the sliding velocity, $h$ is the oil film thickness, and $\dot{\gamma}$ is the shear rate. It should be noted that Eq. (2) is appropriate for Newtonian fluids, and the shear thinning effect is ignored. In fact, the rheological property of lubricating oils significantly changes at high pressure and shear rate conditions, thus the Newtonian fluids would transform to non-Newtonian fluids with a limiting shear stress $\tau_{\text {lim }}$, which is proportional to the hydrodynamic pressure $p_{h}$ [15].

$$
\tau_{\lim }=\mu_{h} p_{h}
$$

where $\mu_{h}$ is the limiting shear stress coefficient that is lubricant specific. Considering the limiting shear stress and shear thinning effect, Eq. (2) can be approximately rewritten by introducing the rheological model proposed by Bair and Winer [16], as follows:

$$
F_{h}=\int_{A_{h}} \tau_{\lim }\left(1-\mathrm{e}^{-\eta \dot{\gamma} / \tau_{\lim }}\right) \mathrm{d} A
$$

For a lubricated point contact, $\eta \dot{\gamma}$ at high hydrodynamic pressure and high shear rate condition is generally far greater than $\tau_{\lim }$, thus the value of $e^{-\eta \dot{\gamma} / \tau_{\text {lim }}}$ is much smaller than 1 . In such a case, Eq. (4)

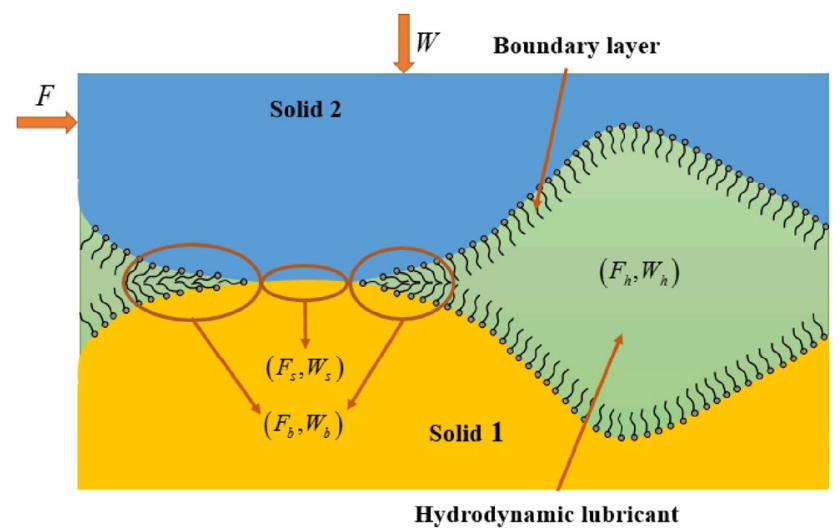

Fig. 2 Schematic of mixed lubrication. can be written as

$$
\begin{aligned}
F_{h} & =\int_{A_{h}} \tau_{\lim }\left(1-\mathrm{e}^{-\eta \dot{\gamma} / \tau_{\text {lim }}}\right) \mathrm{d} A \approx \int_{A_{h}} \tau_{\lim } \mathrm{d} A \\
& =\int_{A_{h}} \mu_{h} p_{h} \mathrm{~d} A=\mu_{h} \int_{A_{h}} p_{h} \mathrm{~d} A=\mu_{h} W_{h}
\end{aligned}
$$

where $W_{h}$ is the hydrodynamic force equated to the integration of hydrodynamic pressure $p_{h} \cdot \mu_{h}$ can also be regarded as a hydrodynamic friction coefficient.

$F_{b}$ and $F_{s}$ are assumed to follow the Amonton's law of friction with a boundary friction coefficient $\mu_{b}$ and a solid-solid contact friction coefficient $\mu_{s}$, respectively

$$
\begin{aligned}
& F_{b}=\mu_{b} W_{b} \\
& F_{s}=\mu_{s} W_{s}
\end{aligned}
$$

where $W_{b}$ is the load fraction supported by the boundary layer and $W_{s}$ is the load fraction supported by real solid-solid contact areas. The total applied load $W$ is balanced by the sum of $W_{h}, W_{b}$ and $W_{s}$ [17], as expressed by Eq. (8).

$$
W=W_{h}+W_{b}+W_{s}
$$

If the two factors, $\alpha$ and $\beta$, are introduced to represent $W_{h}$ as a fraction of the total applied load $W$ and $W_{b}$ as a fraction of the remaining part of applied load $W-W_{h}$ respectively, i.e.,

$$
\begin{gathered}
W_{h}=\alpha W \\
W_{b}=\beta\left(W-W_{h}\right)=\beta(1-\alpha) W \\
W_{s}=(1-\beta)\left(W-W_{h}\right)=(1-\beta)(1-\alpha) W
\end{gathered}
$$

Then, the total friction coefficient can be represented as:

$$
f=\frac{F_{h}+F_{b}+F_{s}}{W}=\mu_{h} \alpha+\mu_{b} \beta(1-\alpha)+\mu_{s}(1-\beta)(1-\alpha)
$$

In general, the solid-solid contact friction coefficient $\mu_{s}$ is higher than the boundary friction coefficient $\mu_{b}$ and the hydrodynamic friction coefficient $\mu_{h}$, and they could be assumed as constants during the running-in process. It is worth noting that the separation of solid-solid contact force $W_{s}$ from other parts 
is meaningful for analyzing not only friction but also wear as discussed in Section 2.2.

\subsection{Estimation of material wear under lubrication condition}

The Archard wear law [9] is a widely used wear equation that has been developed more than 50 years ago. This wear law presumes a linear dependency between the wear volume $V$ of material and total applied normal load $W$, sliding distance $s$ and material hardness $H$ as follows:

$$
V=K \frac{W s}{H}
$$

where $K$ is the wear coefficient.

The wear rate is the derivation of wear volume to time, which can be expressed as:

$$
\dot{V}=\frac{\mathrm{d} V}{\mathrm{~d} t}=K \frac{W}{H} \frac{\mathrm{d} s}{\mathrm{~d} t}=K \frac{W u}{H}
$$

where $u$ is sliding velocity. However, the linear dependency is not always observed in experiments especially at the running-in stage. If the material loss in the hydrodynamic lubricated and boundary layer regions is considered negligible compared to the solid-solid contact case, it is reasonable to assume that material loss occurs only at solid-solid interfaces, thus it depends only on the solid-solid contact force $W_{s}$ rather than the total normal load $W$.

Therefore, the wear rate function can be modified as follows:

$$
\dot{V}=K \frac{W_{s} u}{H}=(1-\beta)(1-\alpha) K \frac{W u}{H}
$$

Conventionally, the effect of hydrodynamic and boundary lubrication on wear rate used to be implicitly incorporated into the value of wear coefficient $K$ because the solid-solid contact force $W_{s}$ is difficult to be estimated in experiments under lubricated test conditions. The above modified wear equation explicitly expresses the effect of hydrodynamic lubrication on wear rate via the term $(1-\alpha)$ and the effect of boundary lubrication via the term $(1-\beta)$, hence the interpretation of wear coefficient $K$ here is different from its conventional meaning. When the hydrodynamic force bears the total normal load $W$ (i.e., $\alpha=1$ ) or the boundary layer fully supports the load of $W-W_{h}$ (i.e., $\beta=1$ ), no wear will occur even if $W$ and $K$ are nonzero. This equation also shows the relationship between the wear volume and friction coefficient $f$ (Eq. (12)) through factors $\alpha$ and $\beta$.

\section{Experimental procedure}

\subsection{Friction measurements}

To analyze the frictional behavior during the running-in process, tribology experiments were carried out on a universal tribometer (Rtec Instrument, USA) that is shown in Fig. 3. Both the changes in surface topography and frictional force during the experimental tests were measured. An ordinary set-up of Rtec tribometer was used for testing, where a steel ball was mounted in the stationary upper sample holder and a lower sample holder with a mounted steel disk performed the reciprocating motion driven by a motor. All tests were set with a load of $20 \mathrm{~N}$, corresponding to a maximum Hertzian contact pressure of approximately 1.0 GPa and a contact radius of approximately $93.8 \mu \mathrm{m}$,

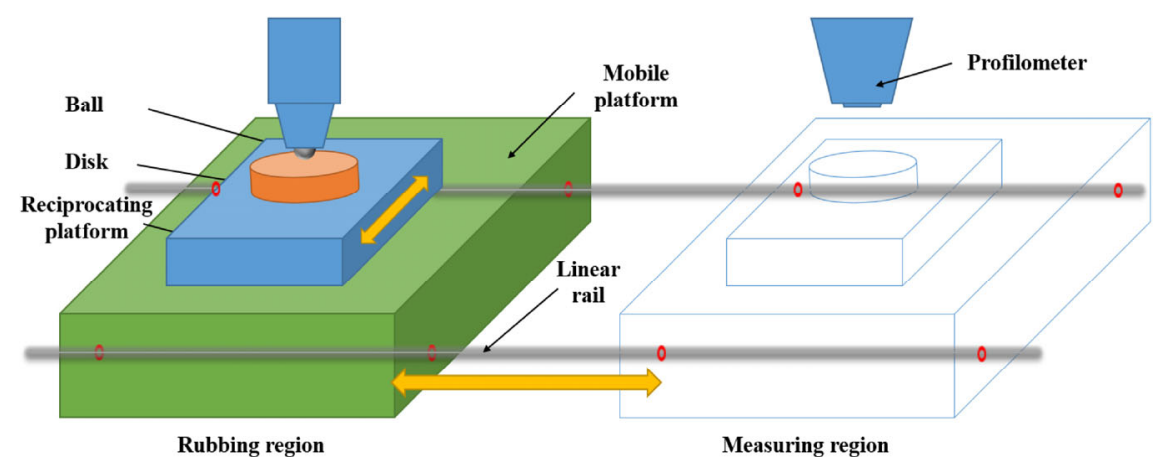

Fig. 3 Schematic of universal tribometer. 
and the sliding speed and sliding amplitude were set to be $20 \mathrm{~mm} / \mathrm{s}$ and $5 \mathrm{~mm}$, respectively.

A start-stop testing scheme was used in order to investigate the evolution of surface topography and distinguish the effect of adsorption boundary layer and surface smoothing caused by mechanical wear on the change in friction during the running-in process. The test is performed according to the following scheme. Every $2 \mathrm{~min}$, the lower holder motor was interrupted and the mobile platform holding the lower specimen was moved from the rubbing region to the measuring region (see Fig. 3) to capture an image of the surface topography, which was measured with a white-light interference profilometer unit attached to the Rtec tribometer. During the interruption period, the lubricant film on the disk specimen was carefully cleaned with acetone before topography measurement. Then the mobile platform was moved back to the rubbing region and the friction test re-started after feeding new lubricant. The start-stop test described above was repeated seven times, and seven images of the worn surface were captured on-site without detaching the test pieces from the holders.

For comparison, a noninterrupted reference experiment with the same load and speed conditions was carried out for a total duration of $14 \mathrm{~min}$. The surfaces of the ball and disk used for all tests were cleaned with acetone in an ultrasonic bath. All tests were performed at $22 \pm 2{ }^{\circ} \mathrm{C}$ under normal atmospheric conditions.

\subsection{Sample preparation}

GCr15 bearing steel ball and disk specimens were used owing to material characteristics of GCr15 bearing steel, including uniform-chemical composition, low percentage of harmful elements, high purity, welldistributed carbide and good surface quality. The $40 \mathrm{~mm}$ diameter disk specimen was finished by grinding with an average roughness $R_{a}$ of $65 \mathrm{~nm}$ and standard deviation (RMS) of $85 \mathrm{~nm}$, measured over an area of $1.819 \mathrm{~mm} \times 1.137 \mathrm{~mm}$ on a white-light interference profilometer. The ball diameter was $12.7 \mathrm{~mm}$ with roughness $R_{a}=6 \mathrm{~nm}$. Base oil POE (Polyol Ester) 32\# (dynamic viscosity at $40{ }^{\circ} \mathrm{C}$ is $0.031 \mathrm{~Pa} \cdot \mathrm{s}$ ) was used as lubricant without additives.

\section{Results and discussion}

\subsection{Comparison of conventional and start-stop friction test results}

Frictional force is relatively easy to be measured continuously in-situ in experiments, and curves of COF plotted against sliding time or distance are typically used to analyze the wear transitions occurred during the experiment [18]. The red line in Fig. 4 shows the measured curve of COF vs. the sliding distance in the nonstop reference experiment. The initial COF value of 0.106 gradually decreased to approximately 0.087 after the running-in stage, representing a typical transition pattern of friction for many lubricated rough surfaces.

The blue line in Fig. 4 is the COF values obtained by the seven start-stop tests. All seven segmental curves show similar monotonic decay trend, and the initial peak of each segment is gradually reduced. The initial COF value obtained by the first start-stop test is 0.107 , which is very close to that obtained by the reference test. The COF values at the end of each segment are also close to those of the reference test, implying that nearly identical running-in transition of friction behavior occurred throughout the friction tests. Based on the common knowledge regarding the running-in process, such gradual decrease in COF values is attributed to the extensive mechanical wear and/or plastic deformation of asperities that leads to

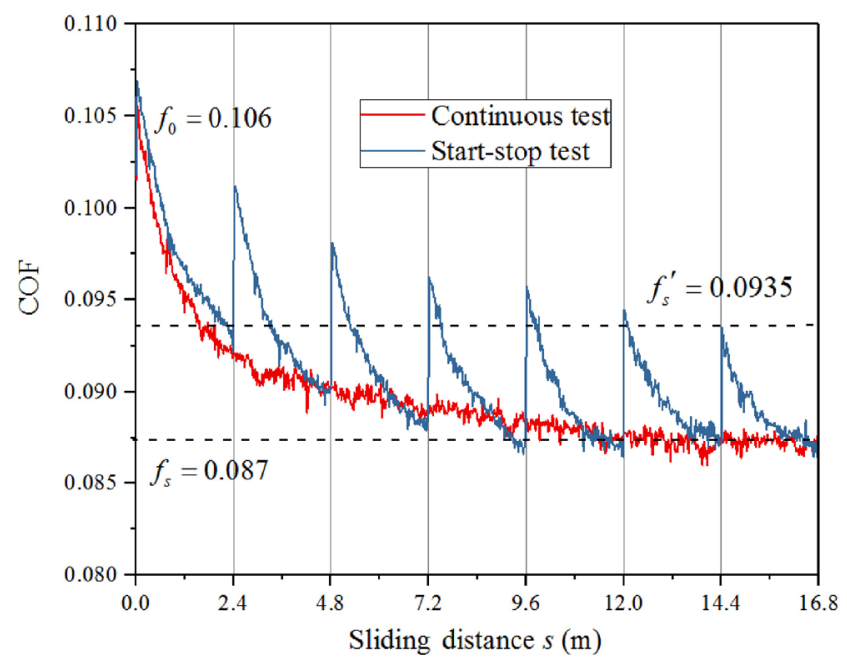

Fig. 4 Comparison of continuous and start-stop friction tests of the same friction pair under lubricated conditions. 
a gradual increase of real contact area and decrease of asperity contact pressure to generate adsorbed boundary layer at asperity contact regions. Meanwhile, the decrease in asperity height enhances the hydrodynamic lubrication of oils reserved in the valleys of rough surface. The COF peak values in the start-stop test are caused by the destruction of adsorbed boundary layer owing to the frequent cleaning at every stop between tests, and the sub-running-in leads to re-formation of the adsorption boundary lubrication layer.

If the above friction test results are re-plotted in a semi-logarithmic coordinate (see Fig. 5), a double trend of friction change for the running-in process can be found for the continuous friction test, as illustrated by the red and green straight lines in Fig. 5(a), while the COF curves for the seven start-stop friction tests are all represented by single straight lines with different slopes. The double trend friction change detected in the continuous friction test implies that two independent processes of surface smoothing and boundary layer formation are involved in the running-in process before the intersection point of the two lines (at sliding distance of approximately $3 \mathrm{~m}$ ). However, the friction change was primarily caused by the surface smoothing due to wear after the intersection point. On the other hand, the startstop friction tests involved combined effect that was shown only in the first and second tests, while the subsequent five tests predominantly involved single process of boundary layer formation. Fig. 5(a) shows that the red straight line is extended to intersect with the vertical axis at approximately 0.097 , denoted as $f_{0}^{\prime}$,

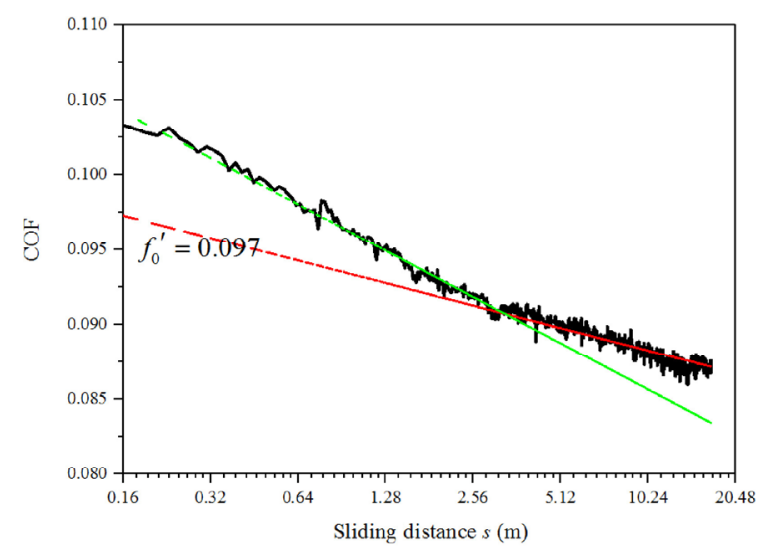

(a) Continuous friction test which is a characteristic parameter of the running-in phase considered to model friction in Section 4.2.

\subsection{Modeling of friction behavior during running-in stage}

Based on the experiment results shown in Figs. 4 and 5 analyzed in Section 4.1, it is postulated that the change in $\mathrm{COF}, f$, during the running-in phase is a function of the hydrodynamic force fraction $\alpha$ and boundary layer force fraction $\beta$. To derive the function of $\mathrm{COF}, f$, the following assumptions are made:

a) The hydrodynamic force fraction $\alpha$ increases from an initial value $\alpha_{0}$ to a steady value $\alpha_{s}$, following Eq. (16) as depicted in Fig. 6(a);

b) The boundary layer force fraction $\beta$ increases from an initial value $\beta_{0}$ to steady value $\beta_{s}$ in a similar manner as shown in Fig. 6(b).

It follows that

$$
-\frac{\mathrm{d} \alpha}{\mathrm{d} s}=k_{\alpha}\left(\alpha-\alpha_{s}\right)
$$

where $k_{\alpha}$ is a rate constant related to the surface smoothing, $s$ is sliding distance.

Then we get

$$
\frac{\mathrm{d}\left(\alpha-\alpha_{s}\right)}{\alpha-\alpha_{s}}=-k_{\alpha} \mathrm{d} s
$$

Thus,

$$
\begin{gathered}
\ln \left(\alpha-\alpha_{s}\right)=-k_{\alpha} s+c \\
\alpha=c^{\prime} e^{-k_{\alpha} s}+\alpha_{s}
\end{gathered}
$$

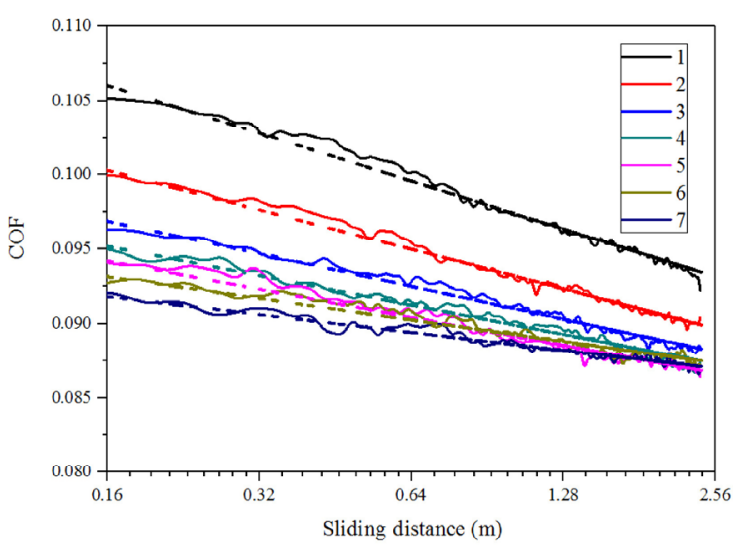

(b) Start-stop friction test

Fig. 5 COF curves of continuous and start-stop friction tests for the same friction pair in semi-logarithmic coordinates. 


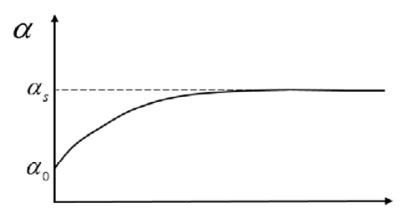

Sliding distance

(a) Change in hydrodynamic bearing fraction $\alpha$

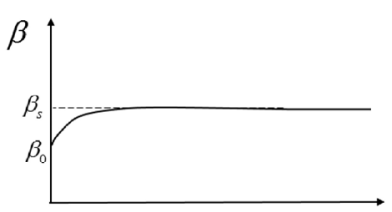

Sliding distance

(b) Change in boundary friction layer bearing fraction $\beta$
Fig. 6 Change in hydrodynamic bearing fraction $\alpha$ and boundary friction layer bearing fraction $\beta$ during running-in process.

When $s=0, \alpha=\alpha_{0}$, therefore, we get $c^{\prime}=\alpha_{0}-\alpha_{s}$

$$
\alpha=\left(\alpha_{0}-\alpha_{s}\right) \mathrm{e}^{-k_{\alpha} s}+\alpha_{s}
$$

Similarly, the boundary layer force fraction can be expressed as

$$
\beta=\left(\beta_{0}-\beta_{s}\right) \mathrm{e}^{-k_{\beta} s}+\beta_{s}
$$

where $k_{\beta}$ is a rate constant related to the formation of boundary layer.

Four key values of the $\operatorname{COF}\left(f_{0}, f_{0}^{\prime}, f_{s}\right.$ and $\left.f_{s}^{\prime}\right)$ are plotted in the Figs. 4 and 5(a). When the changes in $\alpha$ and $\beta$ shown in Fig. 6 are integrated in the model, the four key values of COF can be expressed as follows:

$$
\begin{aligned}
& f_{0}=\mu_{h} \alpha_{0}+\mu_{b} \beta_{0}\left(1-\alpha_{0}\right)+\mu_{s}\left(1-\beta_{0}\right)\left(1-\alpha_{0}\right) \\
& f_{0}^{\prime}=\mu_{h} \alpha_{0}+\mu_{b} \beta_{s}\left(1-\alpha_{0}\right)+\mu_{s}\left(1-\beta_{s}\right)\left(1-\alpha_{0}\right) \\
& f_{s}=\mu_{h} \alpha_{s}+\mu_{b} \beta_{s}\left(1-\alpha_{s}\right)+\mu_{s}\left(1-\beta_{s}\right)\left(1-\alpha_{s}\right) \\
& f_{s}^{\prime}=\mu_{h} \alpha_{s}+\mu_{b} \beta_{0}\left(1-\alpha_{s}\right)+\mu_{s}\left(1-\beta_{0}\right)\left(1-\alpha_{s}\right)
\end{aligned}
$$

or,

$$
\begin{aligned}
f_{0}-f_{0}^{\prime}= & \left(\mu_{b}-\mu_{s}\right)\left(1-\alpha_{0}\right)\left(\beta_{0}-\beta_{s}\right) \\
f_{0}^{\prime}-f_{s}= & \mu_{h}\left(\alpha_{0}-\alpha_{s}\right)-\mu_{b} \beta_{s}\left(\alpha_{0}-\alpha_{s}\right)- \\
& \mu_{s}\left(1-\beta_{s}\right)\left(\alpha_{0}-\alpha_{s}\right) \\
f_{0}-f_{s}^{\prime}= & \mu_{h}\left(\alpha_{0}-\alpha_{s}\right)-\mu_{b} \beta_{0}\left(\alpha_{0}-\alpha_{s}\right)- \\
& \mu_{s}\left(1-\beta_{0}\right)\left(\alpha_{0}-\alpha_{s}\right)
\end{aligned}
$$

Because the coefficient matrix expressed in Eqs. (22)(25) is not of full-rank, the values of $\alpha_{0}, \alpha_{s}, \beta_{0}$ and $\beta_{s}$ cannot be determined by solving Eqs. (22)-(25). It is reasonable to set the value of $\beta_{0}$ to be zero because of the negligible boundary layer formation at the beginning of the test. Given that the magnitudes of $\mu_{h}, \mu_{b}$ and $\mu_{s}$ are known, the three unknown parameters, $\alpha_{0}, \alpha_{s}$ and $\beta_{s}$, can be obtained by solving Eqs. (22)-(25).

As shown in Fig. $4, f_{0}$ is the value of $\mathrm{COF}$ at the onset of the continuous friction test, at which the initial values of both $\alpha$ and $\beta$ are expressed by Eq. (22). $f_{s}$ is the value of COF when the running-in phase is complete with both $\alpha$ and $\beta$ reaching their saturation values. $f_{s}^{\prime}$ is the value of COF when the surface smoothing reached a steady state $\left(\alpha=\alpha_{s}\right)$, while the boundary layer was partially removed $\left(\beta=\beta_{0}\right)$, corresponding to the peak $\mathrm{COF}$ value of the last start-stop friction test. $f_{0}^{\prime}$ shown in Fig. 5(a) represents an imaginary lubrication state where the boundary layer has fully formed $\left(\beta=\beta_{s}\right)$ but the surface smoothing did not occur $\left(\alpha=\alpha_{0}\right)$. All four characteristic values of $\mathrm{COF}, f_{0}, f_{s}, f_{0}^{\prime}$ and $f_{s}^{\prime}$, can be obtained from the experimental results shown in Figs. 4 and 5(a).

Depending on the relative significance of boundary layer formation and surface smoothing to the change in COF during the running-in process, four situations, in which the relationship between COF and sliding distance can be expressed respectively as follows:

Situation A: the formation of adsorbed boundary layer is complete ( $\beta=\beta_{s}$ ), while the surface smoothing continues. In such a case, COF yields:

$$
\begin{aligned}
f= & \mu_{h} \alpha+\mu_{b} \beta_{s}(1-\alpha)+\mu_{s}\left(1-\beta_{s}\right)(1-\alpha) \\
= & \mu_{h} \alpha_{s}+\mu_{b} \beta_{s}\left(1-\alpha_{s}\right)+\mu_{s}\left(1-\beta_{s}\right)\left(1-\alpha_{s}\right)+ \\
& {\left[\mu_{h}\left(\alpha_{0}-\alpha_{s}\right)-\mu_{b} \beta_{s}\left(\alpha_{0}-\alpha_{s}\right)-\right.} \\
& \left.\mu_{s}\left(1-\beta_{s}\right)\left(\alpha_{0}-\alpha_{s}\right)\right] \mathrm{e}^{-k_{\alpha} s} \\
= & f_{s}+\left(f_{0}^{\prime}-f_{s}\right) \mathrm{e}^{-k_{\alpha} s}
\end{aligned}
$$

Situation B: the adsorbed boundary layer has not formed $\left(\beta=\beta_{0}\right)$, while surface smoothing occurs. In this case, $\mathrm{COF}$ becomes:

$$
\begin{aligned}
f= & \mu_{h} \alpha+\mu_{b} \beta_{0}(1-\alpha)+\mu_{s}\left(1-\beta_{0}\right)(1-\alpha) \\
= & \mu_{h} \alpha_{s}+\mu_{b} \beta_{0}\left(1-\alpha_{s}\right)+\mu_{s}\left(1-\beta_{0}\right)\left(1-\alpha_{s}\right)+ \\
& {\left[\mu_{h}\left(\alpha_{0}-\alpha_{s}\right)-\mu_{b} \beta_{0}\left(\alpha_{0}-\alpha_{s}\right)-\right.} \\
& \left.\mu_{s}\left(1-\beta_{0}\right)\left(\alpha_{0}-\alpha_{s}\right)\right] \mathrm{e}^{-k_{\alpha} s} \\
= & f_{0}+\left(f_{0}-f_{s}^{\prime}\right) \mathrm{e}^{-k_{\alpha} s}
\end{aligned}
$$


Situation C: the formation of boundary layer continues, while no mechanical wear occurs $\left(\alpha=\alpha_{0}\right)$ during the running-in process. In this case, the $\mathrm{COF}$ changes with the sliding distance as expressed by Eq. (31).

$$
\begin{aligned}
f= & \mu_{h} \alpha_{0}+\mu_{b} \beta\left(1-\alpha_{0}\right)+\mu_{s}(1-\beta)\left(1-\alpha_{0}\right) \\
= & \mu_{h} \alpha_{0}+\mu_{b} \beta_{s}\left(1-\alpha_{0}\right)+\mu_{s}\left(1-\beta_{s}\right)\left(1-\alpha_{0}\right)+ \\
& \left(\mu_{b}-\mu_{s}\right)\left(1-\alpha_{0}\right)\left(\beta_{0}-\beta_{s}\right) \mathrm{e}^{-k_{\beta} s} \\
= & f_{0}^{\prime}+\left(f_{0}-f_{0}^{\prime}\right) \mathrm{e}^{-k_{\beta} s}
\end{aligned}
$$

Situation D: the formation of boundary layer and surface smoothing occur simultaneously causing a combined effect on the change in COF. Therefore, $f_{0}^{\prime}$ in Eq. (29) should be replaced by Eq. (31). In this general case, the relationship between $\mathrm{COF}, f$, and sliding distance, $s$, is an exponential function, the factor of which also includes an exponential function with a different constant rate $k_{\beta}$ as expressed by Eq. (32).

$$
f=\left[\left(f_{0}-f_{0}^{\prime}\right) \mathrm{e}^{-k_{\beta} s}+f_{0}^{\prime}-f_{s}\right] \mathrm{e}^{-k_{\alpha} s}+f_{s}
$$

Equation (32) transforms into Eq. (29) when the formation of adsorbed boundary film is complete ( $\beta=\beta_{s}$ ) and to Eq. (30) when the adsorbed boundary film does not change $\left(\beta=\beta_{0}\right)$. If the surface smoothing can be negligible during the running-in $\left(\alpha=\alpha_{0}\right)$, Eq. (32) will be simplified as Eq. (31). It worth noting that the derived analytic equations of COF, (Eqs. (29)(32)), depend only on the parameters $f_{0}, f_{s}, f_{0}^{\prime}$, $f_{s}^{\prime}, k_{\alpha}$ and $k_{\beta}$, which can be obtained by friction experiments. Neither the basic properties of $\mu_{h}, \mu_{b}$ and $\mu_{s}$ nor the characteristic fraction parameters, $\alpha_{0}, \alpha_{s}, \beta_{0}$ and $\beta_{s}$ are involved in these analytic friction equations derived in this study.

Regression analysis was performed on the experimental data presented by the red line in Fig. 4 together with the function expressed by Eq. (32), and the obtained values of the rate constants, $k_{\alpha}$ and $k_{\beta}$, for the continuous friction test were $0.24 \mathrm{~m}^{-1}$ and $1.75 \mathrm{~m}^{-1}$, respectively. The regression curve plotted as the green line in Fig. 7 agrees well with the measured results obtained from the continuous friction test. Furthermore, substituting $k_{\alpha}=0.24 \mathrm{~m}^{-1}$ into Eqs. (29) and (30), which expresses the changes in COF with a constant boundary layer force fraction $\beta=\beta_{0}$ and $\beta=\beta_{s}$, respectively, the friction coefficient curves

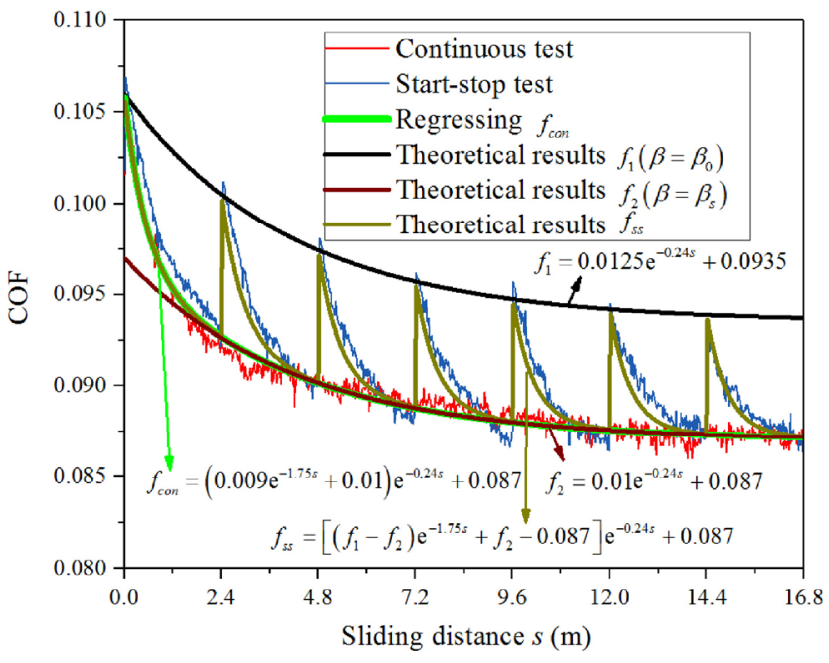

Fig. 7 Comparison of theoretical results and experimental data. Green line is the regression result of continuous test; dark line is the theoretical result of no boundary friction layer.

$f_{1}$ and $f_{2}$ were calculated and their values were plotted as dark and purple lines in Fig. 7. The functions $f_{1}$ and $f_{2}$ can used to express the friction function of the start-stop tests $f_{s \mathrm{~s}}$. It can be seen that the calculated COFs accord well with the experimental values of each start-stop test.

\subsection{Wear-induced changes in surface topography during running-in process}

To explore the evolution of surface roughness under mixed lubrication during the running-in stage, the three-dimensional (3D) topography of wear track formed on the disk specimen was captured at different sliding intervals during the start-stop test series.

Figure 8 shows a set of consequent wear track images. The first image in each column shows the initial (as-manufactured) ground surface. The next seven images represent the grey-scale surface topography captured every two minutes at the same place after each start-stop friction test. When the captured images are compared, it can be seen that the overall grinding marks remain after the running-in stage, implying that the surface was not severely worn; however, wear occurred at the top asperities subjected to direct contact. The change in surface roughness, $R_{a}$, during the running-in process shown in Fig. 9, indicates that the value of $R_{a}$ gradually decreases, which means the surface becomes smoother as the sliding distance increases. 


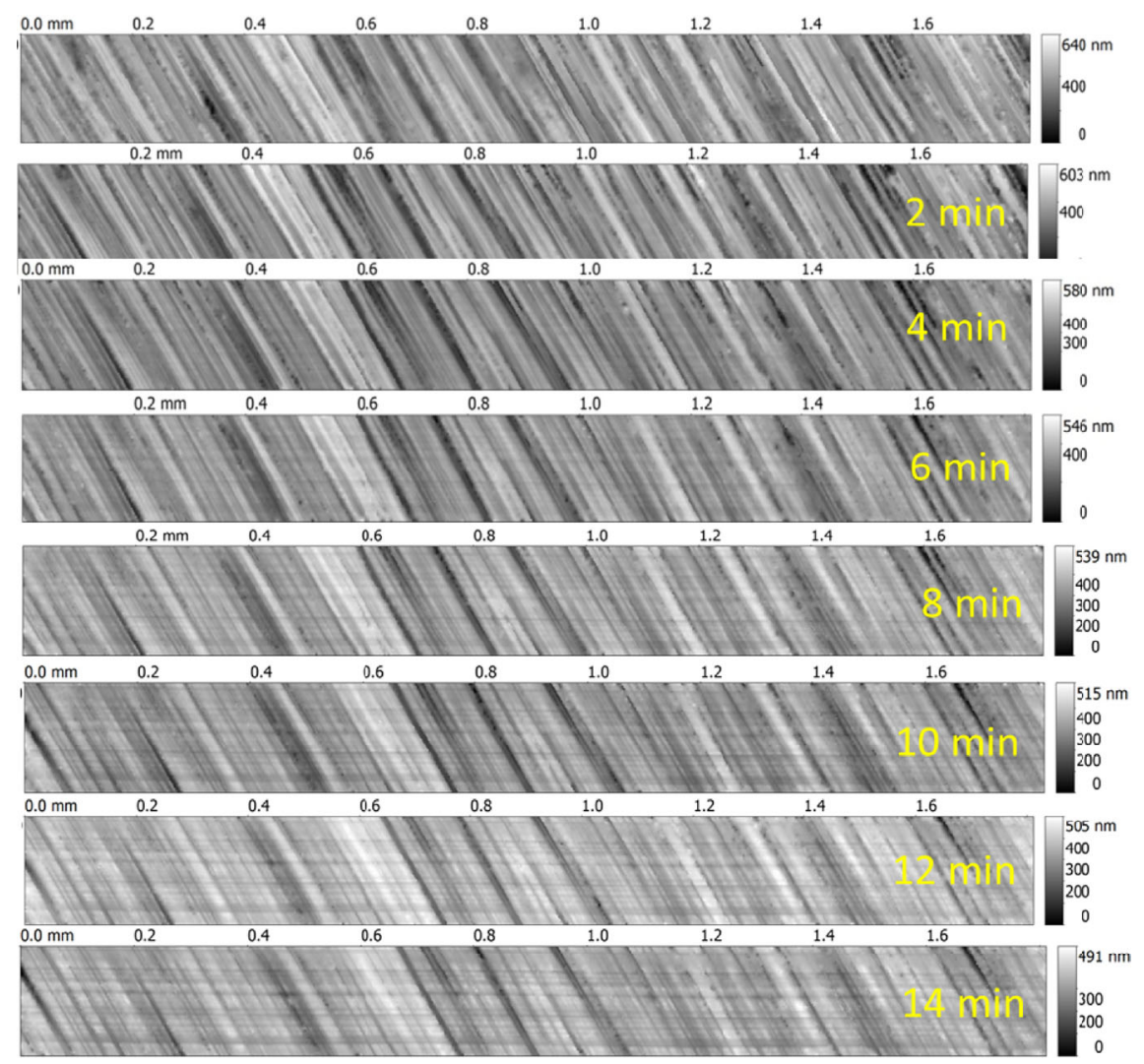

Fig. 8 Change in surface topography with running-in time. Each surface is labeled by corresponding time interval. The rectangular scan size of images corresponds to the wear track size of $1,811 \mu \mathrm{m} \times 195 \mu \mathrm{m}$. The gray scale bar on right side of surface depicts the height range at given time interval.

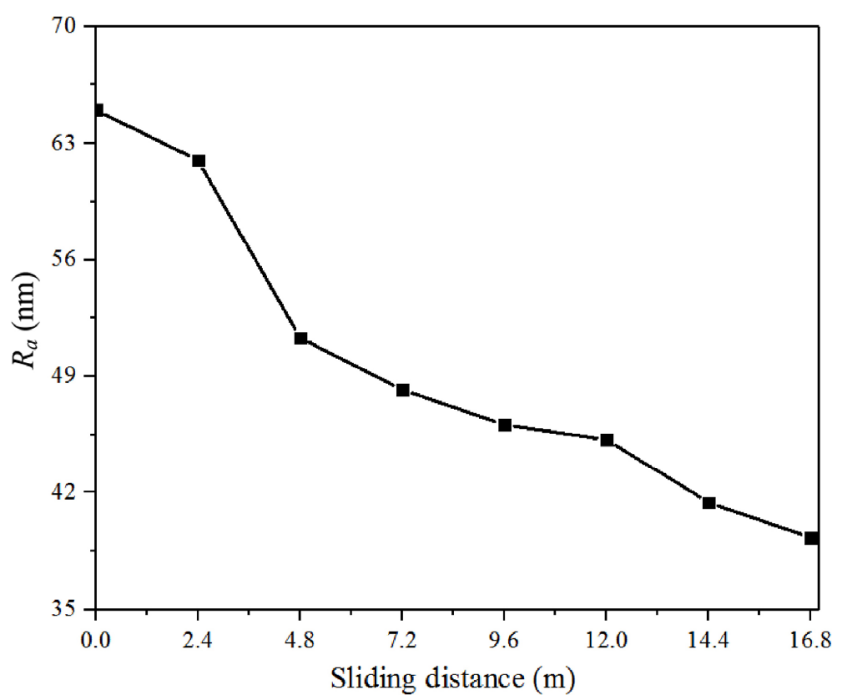

Fig. 9 Change in roughness $R_{a}$ during running-in process.

Compared to Fig. 8, the change in the surface topography during the continuous test shown in Fig. 10 also indicates that the grinding marks are still clearly preserved on the worn surface.
Better understanding of the initial phase of wear during the running-in stage and the accompanying transition phenomena can be gained by continuously evaluating the asperity wear. Researchers in this field $[2,4,5]$ agreed that an equilibrium state between friction surfaces and lubrication is reached after the running-in stage is completed. This balance is characterized by the constant wear rate and constant COF. Based on the friction test result presented in Fig. 4, the steady COF is reached within sliding distance of $12.0 \mathrm{~m}$. This means that the balance between the mechanical response and lubrication condition was reached within this period. The volume loss due to wear will be quantitatively estimated in Section 4.3.1 based on the 3D topography images of subsequent worn surfaces.

\subsubsection{Bearing area curves of material volume for running- in surface}

Volume is a fundamental measure of wear when 


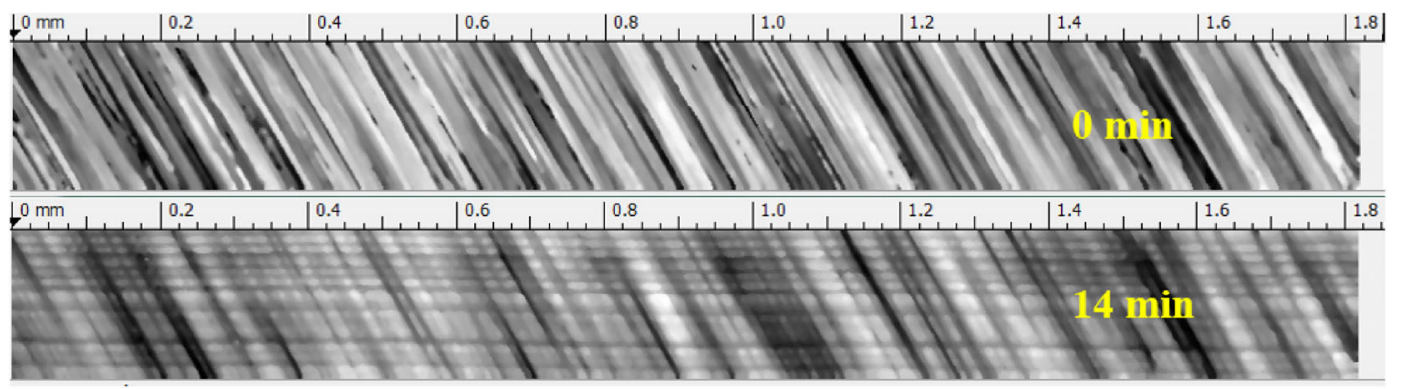

Fig. 10 Change in surface topography during continuous test before and after running-in process.

wear is regarded as the loss of material. Comparative analysis of a surface subjected to wear allows quantitatively estimation of material loss in the case where the same location before and after wear on a surface can be identified exactly [19]. However, this approach is limited by the accuracy of image-processing techniques and reallocation procedure. In this study, the surface transformation due to wear was estimated by comparing the bearing area curves (BAC) instead. This method has a statistical origin and does not depend on the accuracy of reallocation.

Similar to the Abbott-Firestone approach, the BAC method is based on analyzing the functional bearing area curves of investigated surface [20]. Sosa et al. [21] employed the BAC method to monitor the steel surface of ground gears and revealed the distinct difference in height between the initial surface and surfaces subjected to running-in after just 44 cycles. The surface roughness parameters from V-parameter set [22] can be successfully implemented in tribological tasks because the splitting of the material ratio curve into three zones, such as "peak zone", "core zone" and "valley zone", allows distinguishing the changes in worn surface occurred during wear process. The main idea is that the peak zone corresponds to initial running-in wear, the core zone corresponds to wear throughout the lifetime of components, and the valley zone corresponds to lubricant retention under heavy wear conditions. This approach is based on utilizing the surface parameters such as $R \mathrm{pk}, R v \mathrm{k}$, and $R \mathrm{k}$, which were established in Germanic surface geometrical standard DIN 4776 [23]. Furthermore, the areal S-parameter set was defined in ISO 25178-2 [24] and successfully implemented to characterize worn surfaces [25-27]. Yusof et al. [28] has evaluated the blunted asperity peaks of steel surfaces by means of BAC and discussed the applicability of volume parameters $V \mathrm{mp}, V \mathrm{mc}, V_{\mathrm{vc}}$ and $V_{\mathrm{vv}}$ to distinguish the material wear occurred during running-in.

The BAC of a surface is generated by sorting the original sampled surface data in descending order and plotting the sorted data, from 0 to $100 \%$ where $100 \%=N$ (number of data points taken). The BAC considered in this study is a cumulative distribution of material volume. The threshold plane was selected at certain height to calculate the material volume curve as a sum of volumes of single truncated right triangular prisms. The bearing volume curve is crucial for estimating the functional parameters of surfaces on the scale of surface roughness as the volume loss of surface due to wear. The set of material volume BACs, calculated using surface data of each worn surface in Fig. 8, are plotted in Fig. 11.

The bearing area curves of material volume for all time intervals have been plotted as normalized volume data. A height value of BAC is stored in metric term to show the real difference in height occurred due to a wear at the top of asperities during the running-in stage. Because of the evident fact that the "valley zone" of BAC is not disturbed due to wear, the minimum height point is set identical for all curves to arrange the curves according to their height loss in the "peak zone".

Distinct differences between the initial volume (Red line) and all subsequent surfaces can be observed between the peak heights of worn surfaces, as shown in Fig. 11. The Difference in the "core zone" reflects the asperity shape change that caused by plastic deformation. The variance in the curves slope detected in the "core zone" is attributed to the "digitizing error" generated by the light sensor of white-light interferometer device [29]. 


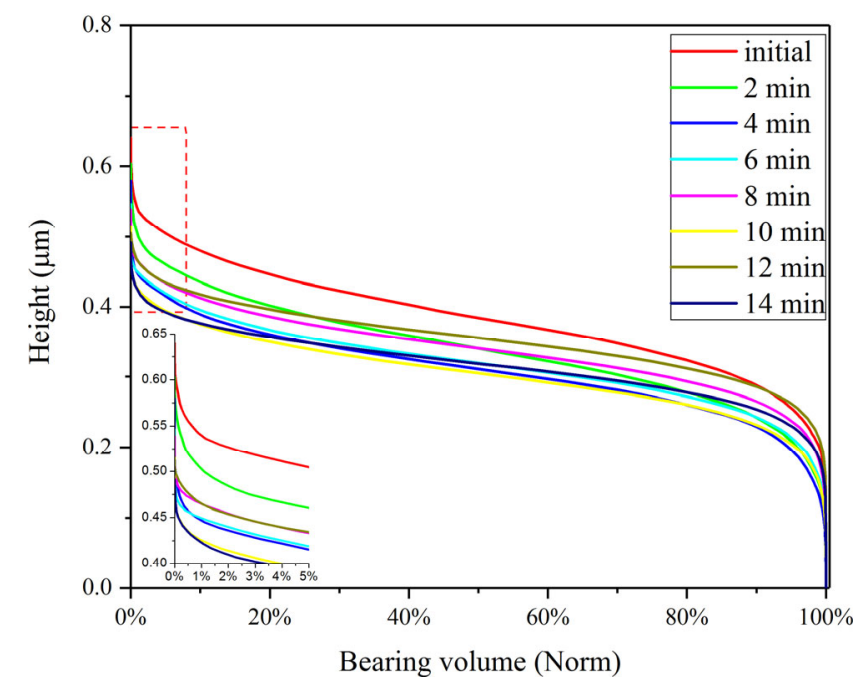

Fig. 11 Bearing curves of material volume calculated based on surface topography data measured every $2 \mathrm{~min}$. Inset-zoomed part (dashed rectangle) of plot shows the overall difference in surface height occurred throughout the test duration time in the short range of $5 \%$.

The surface volume loss at each time interval was estimated as follows: the BAC of initial unworn surface was considered as a reference curve to estimate the volume loss caused by consequent wear. The BAC curve of worn surface has a maximum height lower than the maximum height of the reference curve. This difference is attributed to height loss at asperity peaks. Making the threshold at the maximum height of worn surface regarding to the reference curve, the cutoff part of reference curve, upon the normalized volume axis, is accepted as a plausible material loss due to wear. The obtained values of material loss at each time interval were used in the quantitative analysis of running-in wear discussed in Section 4.3.2.

\subsubsection{Wear characteristics of surface subjected to running-in}

The estimated wear volumes were plotted against the sliding distance and indicated by square marks in Fig. 12. Unlike the predictions made based on Archard's wear model [9], the fitted plot of experimental data shows that the wear volume tends to increase nonlinearly as the sliding distance increases. However, these findings are consistent with the common knowledge in this domain.

The wear rate plotted against sliding distance is evaluated in Fig. 13 (Dark scatter and dash line). The

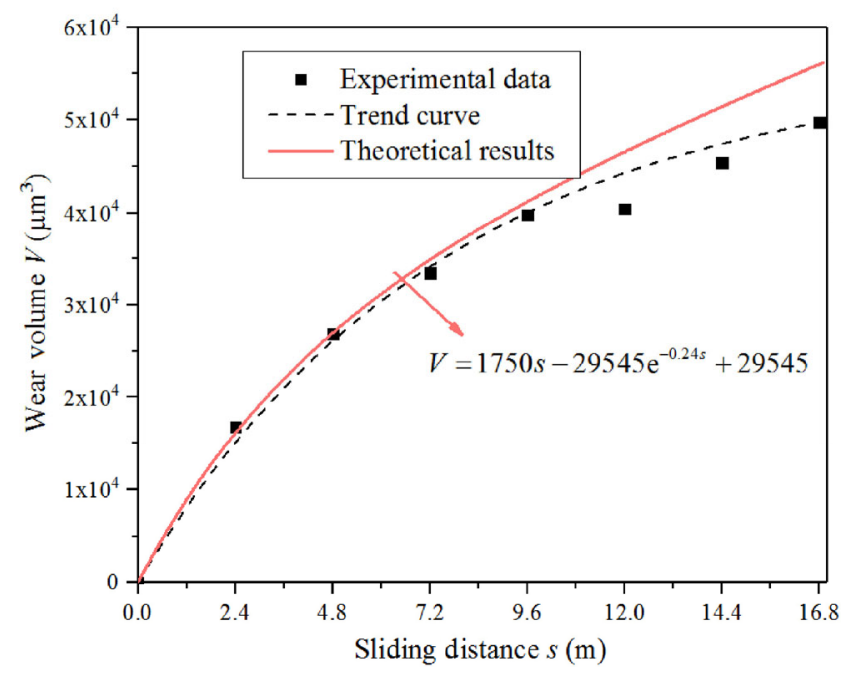

Fig. 12 Evolution of wear volume. Material volume loss at the top of asperities gradually stabilizes with the increase in sliding distance.

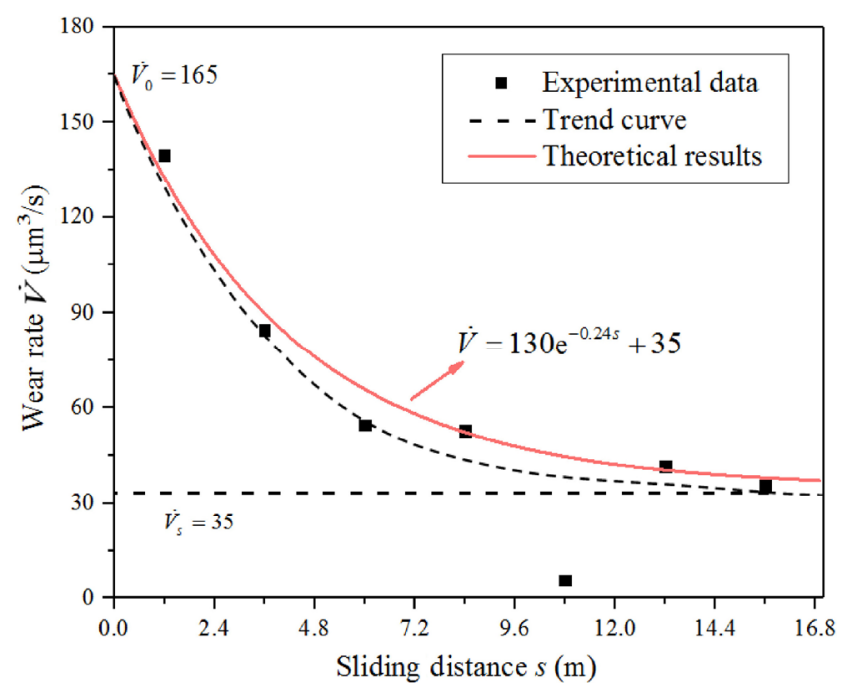

Fig. 13 Evaluation of wear rate against sliding distance. The wear rate was calculated as a ratio of material loss and wear time.

initial wear rate is high because of the small actual contact area and high local pressure. After a certain period of running-in, the surface asperities gradually flatten and the mean pressure of asperity contact decreases, resulting in lower wear rate. Next, an analytical model of wear rate during running-in will be established based on the following assumptions:

(a) The reduction in wear rate is caused by the surface smoothing effect during running-in, and the formation of boundary friction layer reoccurs in each interval;

(b) The hydrodynamic force fraction $\alpha$ increases 
from $\alpha_{0}$ to $\alpha_{s}$ as shown in Fig. 6(a);

(c) Because the boundary layer is regenerated in each test interval, the boundary layer force fraction $\beta$ was assumed as a mean value $\bar{\beta}$ for each interval.

Based on Eq. (15), wear rate can be expressed as:

$$
\begin{aligned}
\dot{V} & =(1-\bar{\beta})(1-\alpha) K \frac{W u}{H} \\
& =(1-\bar{\beta})\left\{1-\left[\left(\alpha_{0}-\alpha_{s}\right) \mathrm{e}^{-k_{\alpha} s}+\alpha_{s}\right]\right\} K \frac{W u}{H} \\
& =(1-\bar{\beta})\left(1-\alpha_{s}\right) K \frac{W u}{H}+(1-\bar{\beta})\left(\alpha_{s}-\alpha_{0}\right) K \frac{W u}{H} \mathrm{e}^{-k_{\alpha} s}
\end{aligned}
$$

where $u$ is the sliding speed. The initial wear rate can be written as:

$$
\dot{V}_{0}=(1-\bar{\beta})\left(1-\alpha_{0}\right) K \frac{W u}{H}
$$

The steady wear rate can be expressed as:

$$
\dot{V}_{s}=(1-\bar{\beta})\left(1-\alpha_{s}\right) K \frac{W u}{H}
$$

Thus, we can obtain:

$$
\dot{V}_{0}-\dot{V}_{s}=(1-\bar{\beta})\left(\alpha_{0}-\alpha_{s}\right) K \frac{W u}{H}
$$

During the running-in process, the wear rate changes with sliding distance and can be written as:

$$
\begin{aligned}
\dot{V} & =(1-\bar{\beta})\left(1-\alpha_{s}\right) K \frac{W u}{H}+(1-\bar{\beta})\left(\alpha_{s}-\alpha_{0}\right) K \frac{W u}{H} \mathrm{e}^{-k_{\alpha} s} \\
& =\dot{V}_{s}+\left(\dot{V}_{0}-\dot{V}_{s}\right) \mathrm{e}^{-k_{\alpha} s}
\end{aligned}
$$

Wear volume is the integral of wear rate to the time, thus it can be expressed as:

$$
\begin{aligned}
V & =\int_{0}^{t} \dot{V} \mathrm{~d} t=\int_{0}^{t} \dot{V}_{s}+\left(\dot{V}_{0}-\dot{V}_{s}\right) \mathrm{e}^{-k_{\alpha} s} \mathrm{~d} t \\
& =\int_{0}^{t} \dot{V}_{s}+\left(\dot{V}_{0}-\dot{V}_{s}\right) \mathrm{e}^{-k_{\alpha} u t} \mathrm{~d} t \\
& =\frac{\dot{V}_{s}}{u} s+\frac{\left(\dot{V}_{0}-\dot{V}_{s}\right)}{k_{\alpha} u}-\frac{\left(\dot{V}_{0}-\dot{V}_{s}\right)}{k_{\alpha} u} \mathrm{e}^{-k_{\alpha} s}
\end{aligned}
$$

Equations (37) and (38) analytically express the changes in wear rate and wear volume with the increase in sliding distance, respectively. The predicted results obtained based on Eqs. (37) and (38) are indicated by red lines in Figs. 12 and 13, respectively. It is found that reasonably accurate predictions of wear rate and wear volume can be obtained by the analytical models compared to the experimental results. Furthermore, when Eqs. (37) and (32) are compared, a conjunctive relationship between friction and wear rate can be detected during running-in. When $s \rightarrow \infty$, $\dot{V} \rightarrow \dot{V}_{s}$ and $f \rightarrow f_{s}$ indicate that COF and wear rate change and attain constant value simultaneously, which is consistent with the universally accepted explanation of friction and wear behaviors during running-in process.

\section{Conclusions}

Analytical equations of $\mathrm{COF}$ and wear rate have been derived considering the combined effect of boundary layer formation and asperity flattening during the running-in process of rough metallic surfaces under lubricated conditions. The findings indicate that the reduction in COF is primarily caused by the combined effect of the adsorbed boundary layer and adaptive surface smoothing due to mechanical wear. This effect could be a combination of dissipative processes at the interface, and it must be analyzed within the context of the given tribosystem and operating conditions.

During the running-in stage, the adsorbed boundary layer generates fast $(3.0 \mathrm{~m})$ and surface smoothing can last for longer sliding distance $(12.0 \mathrm{~m})$. For the start-stop tests, the adsorbed boundary layer regenerates every time after each stop, thus the running-in occurs in every test. The analytical equations derived to calculate the $\mathrm{COF}$ can adequately describe the change in COF during running-in when compared to the experimental results.

The BACs generated from the 3D topography images captured in consequential start-stop test series allow estimating the material loss and wear rate during running-in. The results show that the material loss increases nonlinearly with the increase in sliding distance at a gradually reduced wear rate. Analytical models to describe the material wear volume and wear rate during running-in have been proposed, and the results obtained by these models agree well with the experimental data. The analytical models proposed 
in this study to estimate friction and wear rate are conjunctive, which accords with common knowledge in this domain.

\section{Acknowledgements}

This work was partially supported by NSFC under grant No. 51635009 and by the State Administration of Foreign Expert Affairs under grant No. DL2017QHDX001.

Open Access: The articles published in this journal are distributed under the terms of the Creative Commons Attribution 4.0 International License (http:// creativecommons.org/licenses/by/4.0/), which permits unrestricted use, distribution, and reproduction in any medium, provided you give appropriate credit to the original author(s) and the source, provide a link to the Creative Commons license, and indicate if changes were made.

\section{References}

[1] Blau P J. Running-in. In Encyclopedia of Tribology. Wang Q J, Chung Y W, Eds. Boston, MA: Springer, 2013: 2967-2969.

[2] Blau P J. On the nature of running-in. Tribol Int 38(11-12): 1007-1012 (2005)

[3] Williams J. Engineering Tribology. Cambridge (UK): Cambridge University Press, 2005.

[4] Blau P J. Mechanisms for transitional friction and wear behavior of sliding metals. Wear 72(1): 55-66 (1981)

[5] Blau P J. How common is the steady-state? The implications of wear transitions for materials selection and design. Wear 332-333: 1120-1128 (2015)

[6] Blau P J. Embedding wear models into friction models. Tribol Lett 34(1): 75-79 (2009)

[7] Goryacheva I G. Wear models. In Contact Mechanics in Tribology. Goryacheva I G, Ed. Dordrecht: Springer, 1998: 163-190.

[8] Meng H C, Ludema K C. Wear models and predictive equations: their form and content. Wear 181-183: 443-457 (1995)

[9] Archard J. Contact and rubbing of flat surfaces. J Appl Phys 24(8): 981-988 (1953)

[10] Bowden F P, Leben L. The friction of lubricated metals. Philos Trans R Soc A Math Phys Eng Sci 239(799): 1-27 (1940)
[11] Bai L Q, Meng Y G, Khan Z A, Zhang V. The synergetic effects of surface texturing and MoDDP additive applied to ball-on-disk friction subject to both flooded and starved lubrication conditions. Tribol Lett 65(4): 163 (2017)

[12] Raymond G B. Mechanical Wear Fundamentals and Testing. 2nd ed. New York (USA): Marcel Dekker Inc., 2004.

[13] Kapoor A, Williams J A, Johnson K L. The steady state sliding of rough surfaces. Wear 175(1-2): 81-92 (1994)

[14] Straffelini G. Wear processes. In Friction and Wear: Methodologies for Design and Control. Straffelini G, Ed. Cham: Springer, 2015: 115-158.

[15] Bair S S. High Pressure Rheology for Quantitative Elastohydrodynamics. Amsterdam (Netherlands), Boston (USA): Elsevier, 2007.

[16] Bair S, Winer W O. A rheological model for elastohydrodynamic contacts based on primary laboratory data. J Lubr Technol 101(3): 258-264 (1979)

[17] Hao L C, Meng Y G. Numerical prediction of wear process of an initial line contact in mixed lubrication conditions. Tribol Lett 60(2): 31 (2015)

[18] Bhushan B, Israelachvili J N, Landman U. Nanotribology: friction, wear and lubrication at the atomic scale. Nature 374(6523): 607-616 (1995)

[19] Wang W, Wong P L. Wear volume determination during running-in for PEHL contacts. Tribol Int 33(7): 501-506 (2000)

[20] Abbott E J, Firestone F A. Specifying surface quality. Mech Eng, 55: 569-572 (1933)

[21] Sosa M, Sellgren U, Björklund S, Olofsson U. In situ running-in analysis of ground gears. Wear 352-353: 122-129 (2016)

[22] Jiang X, Scott P J, Whitehouse D J, Blunt L. Paradigm shifts in surface metrology. Part II. The current shift. Proc R Soc A Math Phys Eng Sci, 463(2085): 2071-2099 (2007)

[23] DIN 4776. Kenngrößen Rk, Rpk, Rvk, Mr1, Mr2 zur Beschreibug des Materialanteils im RauheitsprofilMeßbedingungen und Auswerteverfahren. In Deutsche Norm. Berlin: Beuth Verlag GmbH, 1990.

[24] ISO. ISO 25178-2: 2012 Geometrical Product Specifications GPS) - Surface texture: Areal - Part 2: Terms, definitions and surface texture parameters. ISO, Geneva, 2012.

[25] Böhm H J. Parameters for evaluating the wearing behaviour of surfaces. Int J Mach Tools Manu 32(1-2): 109-113 (1992)

[26] Corral I B, Calvet J V, Salcedo M C. Use of roughness probability parameters to quantify the material removed in plateau-honing. Int J Mach Tools Manu 50(7): 621-629 (2010) 
[27] Franco L A, Sinatora A. 3D surface parameters (ISO 25178-2): Actual meaning of $S_{p k}$ and its relationship to $V_{m p}$. Precis Eng 40: 106-111 (2015)

[28] Yusof N F M, Ripin Z M. A technique to measure surface asperities plastic deformation and wear in rolling contact.

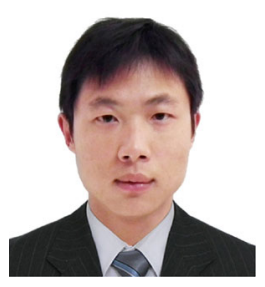

Yazhao ZHANG. He received his bachelor degree in agricultural mechanization and automation in 2014 from Jilin University, Changchun,

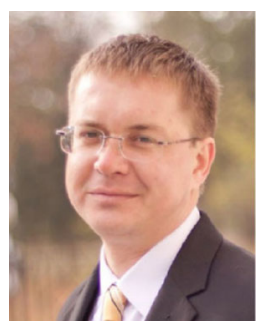

Alexander KOVALEV. He received his M.S. degree in physics from the Gomel State University, Gomel, Belarus in 1997. He has earned PhD degree in tribology and physics of solids in 2007 from Metal-Polymer

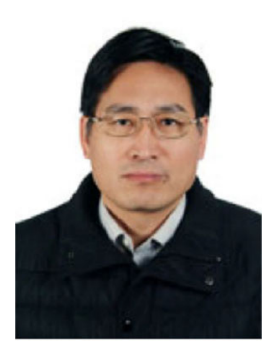

Yonggang MENG. He received his M.S. and $\mathrm{PhD}$ degrees in mechanical engineering from Kumamoto University, Japan, in 1986 and 1989, respectively. He joined the State Key Laboratory of Tribology at
Wear 368-369: 496-504 (2016)

[29] Podulka P, Pawlus P, Dobrzański P, Lenart A. Spikes removal in surface measurement. J Phys Conf Ser 483(1): 012025 (2014)

China. After then, he was a PhD student in the State Key Laboratory of Tribology at Tsinghua University, Beijing, China. His research interests include mixed lubrication and running-in process.

Research Institute, Gomel, Belarus. His current position is a research fellow at State Key Laboratory of Tribology at Tsinghua University, China. His research focuses on micro/nano-tribology, contact mechanics, interfacial phenomena, surface characterization, etc.

Tsinghua University from 1990. His current position is a professor and the director of the laboratory. His research areas cover the tribology of MEMS and hard disk drives, active control of friction and interfacial phenomena and nanomanufacturing. 